7 - ORIGINAL ARTICLE

CLINICAL INVESTIGATION

\title{
Progressive loss of E-cadherin immunoexpression during cervical carcinogenesis ${ }^{1}$
}

\author{
José Roosevelt Cavalcante', João Paulo Aguiar Sampaio"I, João Tarcísio Alves Maia \\ Filho ${ }^{\text {III }}$, Renato Braga Vieira ${ }^{\text {III }}$, José Eleutério Júnior ${ }^{\text {IV }}$, Roberto César Pereira Lima \\ Júniorv, Ronaldo Albuquerque Ribeiro ${ }^{\mathrm{VI}}$, Paulo Roberto Carvalho Almeida ${ }^{\mathrm{VII}}$
}

DOI: http://dx.doi.org/10.1590/S0102-8650201400160007

IFellow Master degree, Postgraduate Program in Pathology, Department of Pathology and Forensic Medicine, Assis Chateaubriand Hospital and School Maternity, Federal University of Ceara (UFC), Fortaleza-CE, Brazil. Technical procedures, acquisition and interpretation of data, statistical analysis, manuscript writing.

IIFellow Master degree, Postgraduate Program in Pathology, Department of Pathology and Forensic Medicine, UFC, Fortaleza-CE, Brazil. Acquisition of data, technical procedures.

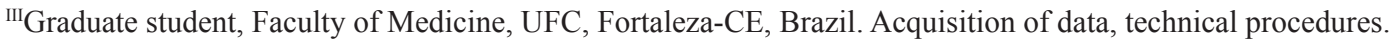

${ }^{\mathrm{IV}} \mathrm{PhD}$, Associate Professor, Department of Maternal and Child Health, UFC, Fortaleza-CE, Brazil. Interpretation of data, manuscript writing.

vPhD, Associate Professor, Department of Physiology and Pharmacology, UFC, Fortaleza-CE, Brazil. Interpretation of data, manuscript writing, statistical analysis, final approval of the manuscript.

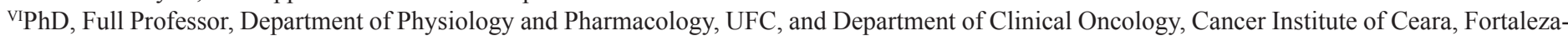
CE, Brazil. Recipient of a CNPq Research Productivity Fellowship, level 1A. Design of the study, interpretation of data, statistical analysis, final approval of the manuscript.

VIIPhD, Associate Professor, Department of Pathology and Forensic Medicine, Master of Science in Pathology, UFC, Fortaleza-CE, Brazil. Conception, design, intellectual and scientific content of the study; critical analysis; final approval of the manuscript.

\section{ABSTRACT}

PURPOSE: To investigate E-cadherin immunoexpression during cervical carcinogenesis.

METHODS: We assessed the immunohistochemical expression of E-cadherin in squamous intraepithelial lesions (SIL - 52 cases), squamous cell carcinoma (SCC) of the uterine cervix (23 cases) and also in eight cases of cervicitis.

RESULTS: The results show very different E-cadherin membrane expression levels when cervicitis (88\%), SILs (73\%) and SCC (17\%) were compared. In SILs, higher E-cadherin loss was seen in less differentiated cells in the basal third of the epithelium. This study suggests that the absence of E-cadherin expression in the membrane is a molecular event that is observed more often in SCC of the uterine cervix than in SILs or cervicitis.

CONCLUSIONS: E-cadherin is an essential molecule during the process of cervical carcinogenesis and in this context exhibits a different expression pattern according to the epithelial thickness layer.

Key words: Cadherins. Immunohistochemistry. Cervical Intraepithelial Neoplasia. Neoplasms squamous cell. Uterine Cervical Neoplasms. 


\section{Introduction}

Cervical cancer is one of the most frequently occurring malignant neoplasms worldwide and is responsible for high mortality rates. It is ranked as the fourth most common carcinoma among women in developing countries. Approximately 528,000 new cases were estimated for 2012, and 266,000 deaths were recorded during the same period ${ }^{1}$. Among cervical carcinomas, squamous cells carcinoma (SCC) represents the most common histological type, accounting for $85 \%$ to $90 \%$ of cases. In 2014, Brazilian estimates predict the occurrence of 15,590 new cases, which indicates an approximate risk of 15.33 cases per 100,000 women ${ }^{2}$.

Currently, it is thought that virtually all cervical cancers are related to human papillomavirus (HPV) infections by one of the 14 types considered to exhibit high oncogenic risk, among which the most common are HPV16 and HPV18,3. Cervical squamous cell carcinoma has well-defined clinical stages and represents a good model for investigating the carcinogenic process ${ }^{5,6}$. It begins with pre-existing non-invasive squamous precursor lesions such as cervical intraepithelial neoplasia (CINs) or squamous intraepithelial lesions (SILs), which are histologically classified according to the degree of involvement of epithelial thickness ${ }^{7-9}$.

The full spectrum of carcinogenic lesions that occurs in a normal cervix includes Low-Grade Squamous Intraepithelial Lesions (LSIL), High-Grade Squamous Intraepithelial Lesions (HSIL) (formerly called dysplasia or cervical intraepithelial neoplasia) and carcinoma in situ, whereas tumor progression is composed of locally invasive squamous cell carcinoma and distant metastatic lesions. Among these steps, the spread of the tumor is the major cause of treatment failure and mortality in women with this type of cancer ${ }^{5,6}$.

According to Hanahan and Weinberg ${ }^{10}$, metastases are responsible for $90 \%$ of human cancer deaths. During metastatic progression, polarized epithelial cancer cells acquire invasive migratory characteristics. This phenotypic conversion allows tumor cells to dissociate from the original tissue and spread to reach distant organs. This type of epithelial cell plasticity is associated with the loss of epithelial traits and the gain of mesenchymal characteristics and a migratory phenotype through a process known as the epithelial-mesenchymal transition (EMT) $)^{5,11}$.

In squamous epithelia, E-cadherin is an essential caretaker of the epithelial phenotype and is one of the main proteins constituting the intercellular adhesion zone that defines tissue architecture and differentiation ${ }^{12}$. Loss of E-cadherin is a common feature of EMT and is associated with a majority of epithelial cancers ${ }^{13}$. In fact, Behrens et $a .^{14}$ have demonstrated that epithelial cells assume invasive characteristics as a result of the loss of E-cadherin-mediated cell adhesion. Burdsal, Damsky and Pedersen ${ }^{15}$ have shown that blocking E-cadherin is sufficient to trigger EMT in mammalian cell systems. Indeed, a downregulation of E-cadherin is frequently associated with strong invasive tendencies and can be considered a classical marker of poor prognosis ${ }^{16}$.

Some laboratories have investigated the biological and morphological characteristics of invasive cancer cells in blocks of cell aggregates. The core cells of these infiltrating tumors appear to have features that are distinct from the cells located at the invasion front, where single cells or small cell clusters (up to five cells) exhibiting increased invasive potential can be found ${ }^{17}$. Furthermore, when these cells are separated from the main tumor mass, they can invade the stroma ${ }^{18}$. These cell groups, which are designated as tumor budding cells, are easily identified by hematoxylin-eosin (HE) staining ${ }^{19}$, and studies have shown a relationship between the presence of these cells and the patient's prognosis ${ }^{19-21}$. It is thought that tumor budding involves two independent processes: loss of cellular cohesion and high migratory characteristics ${ }^{17}$. Most likely, a strong relationship exists among tumor buds, EMT and E-cadherin down-regulation ${ }^{11,22}$.

Thus, this study aims to compare E-cadherin immunoexpression in cervical SILs, SCCs and cervicitis.

\section{Methods}

A cross-sectional study was performed on 83 cases of cervical biopsies and conizations obtained from the archives of the Department of Pathology and Forensic Medicine, Federal University of Ceara (Brazil). The following diagnoses were represented: LSIL (24), HSIL (28), SCC (23 cases) and cervicitis (eight cases). Patient age ranged from 19 to 78 years, with a mean and standard deviation for each group of $29 \pm 7,35 \pm 11,50 \pm 14$ and $25 \pm 8$ years old, respectively.

Histological sections $3 \mu \mathrm{m}$ thick were generated and subsequently applied to silanized slides. Afterwards, antigen retrieval was performed. Next, the slides were incubated with primary antibody (Monoclonal Mouse Anti-Human E-cadherin - Clone NCH-38, Dako North America, Inc, Carpinteria, CA, USA.), and the reaction was amplified. Diaminobenzidine (DABDako) was applied for 10 minutes and was used as the chromogenic substrate. Hematoxylin (Hematoxylin EnVision Flex) was used for nuclear counterstaining. Finally, the slides were mounted and analyzed by microscopy. All phases of the immunohistochemical procedure included samples for positive and negative controls. The cecal appendix was used as the positive control ${ }^{23,24}$. For the negative control, no primary antibody was used. The cases that 
exhibited cell membrane staining for E-cadherin were considered positive, and the absence of membrane expression was interpreted as negative ${ }^{25,26}$. The software GraphPad Prism 5 was utilized for the statistical tests and the construction of graphics. Fisher's exact test was used to assess contingency tables, and $p<0.05$ was defined as statistically significant. The ethics committee of the School Maternity Assis Chateaubriand of the Federal University of Ceara approved this study under protocol number 91/2011.

\section{Results}

In Table 1, we show that cervicitis and squamous intraepithelial lesions SCCs) exhibit a marked predominance of positive E-cadherin staining (cervicitis $=88 \%$; LSILs $=71 \%$; HSILs $=75 \%$, Figure $1(\mathrm{C}-\mathrm{H})$ ). Panels A and B (Figure 1) represent the positive and negative controls, respectively. In contrast, negative expression prevails in SCCs (83\%), (Figure 2 C, D, E). Figure 2A represents SCC E-cadherin negative immunoexpression with contiguous HSIL exhibiting positive expression. A higher magnification of the detailed area shown in Figure 2, panel A is shown in Figure 2B.

TABLE 1 - E-cadherin immunoexpression in Cervicitis, Squamous Intraepithelial Lesions (SIL) and Squamous Cell Carcinoma (SCC) of the uterine cervix.

\begin{tabular}{lccc}
\hline \multirow{2}{*}{ Lesion } & \multicolumn{2}{c}{ E-cadherin expression } & \multirow{2}{*}{ TOTAL } \\
\cline { 2 - 3 } & Negative & Positive & \\
\hline Cervicitis & $1(12 \%)^{*}$ & $7(88 \%)$ & 8 \\
LSIL & $7(29 \%)$ & $17(71 \%)$ & 24 \\
HSIL & $7(25 \%)$ & $21(75 \%)$ & 28 \\
SCC & $19(83 \%)^{*}$ & $4(17 \%)$ & 23 \\
TOTAL & & & 83 \\
\hline
\end{tabular}

$* \mathrm{p}=0.0009$
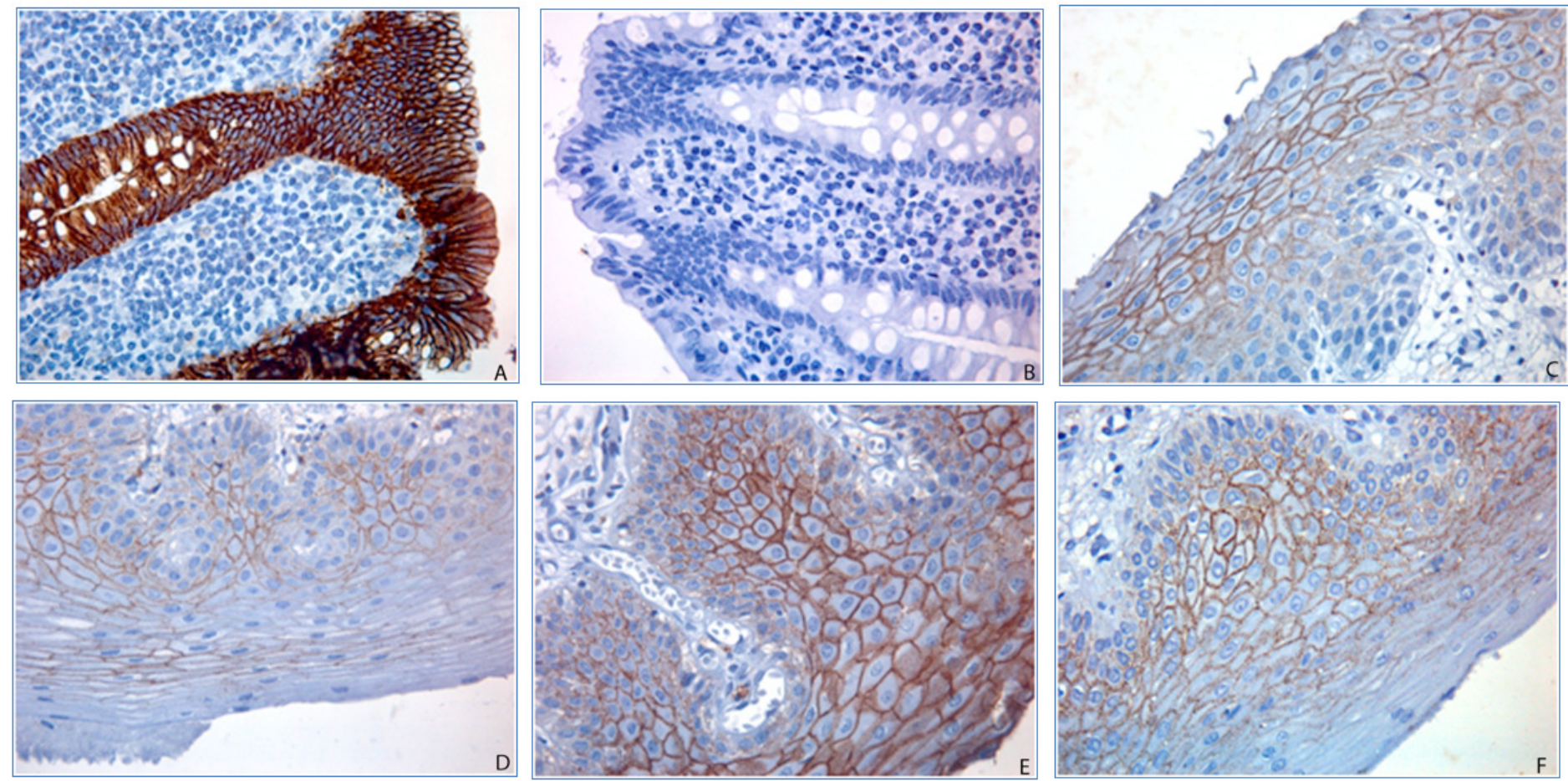
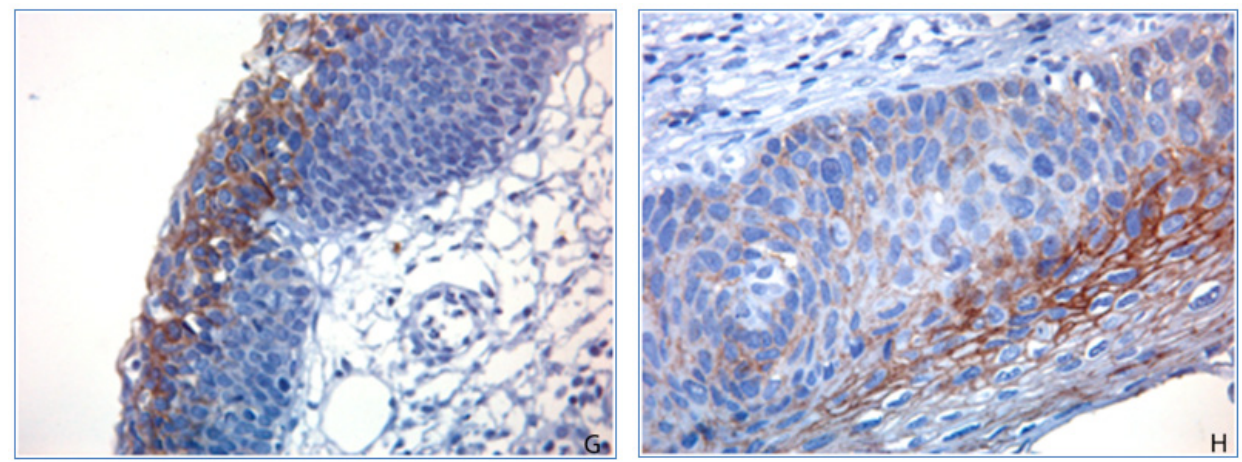

FIGURE 1 - E-Cadherin Immunoexpression in cervicitis and in SILs: (A) Cecal appendix - positive control; (B) Negative control; (C) and (D) Cervicitis - intermediate third positive; (E) LSIL - intermediate and superficial thirds positive; (F) LSIL - intermediate third positive; (G) HSIL intermediate third negative; (H) HSIL - intermediate third positive (A-H, x400 magnification). 

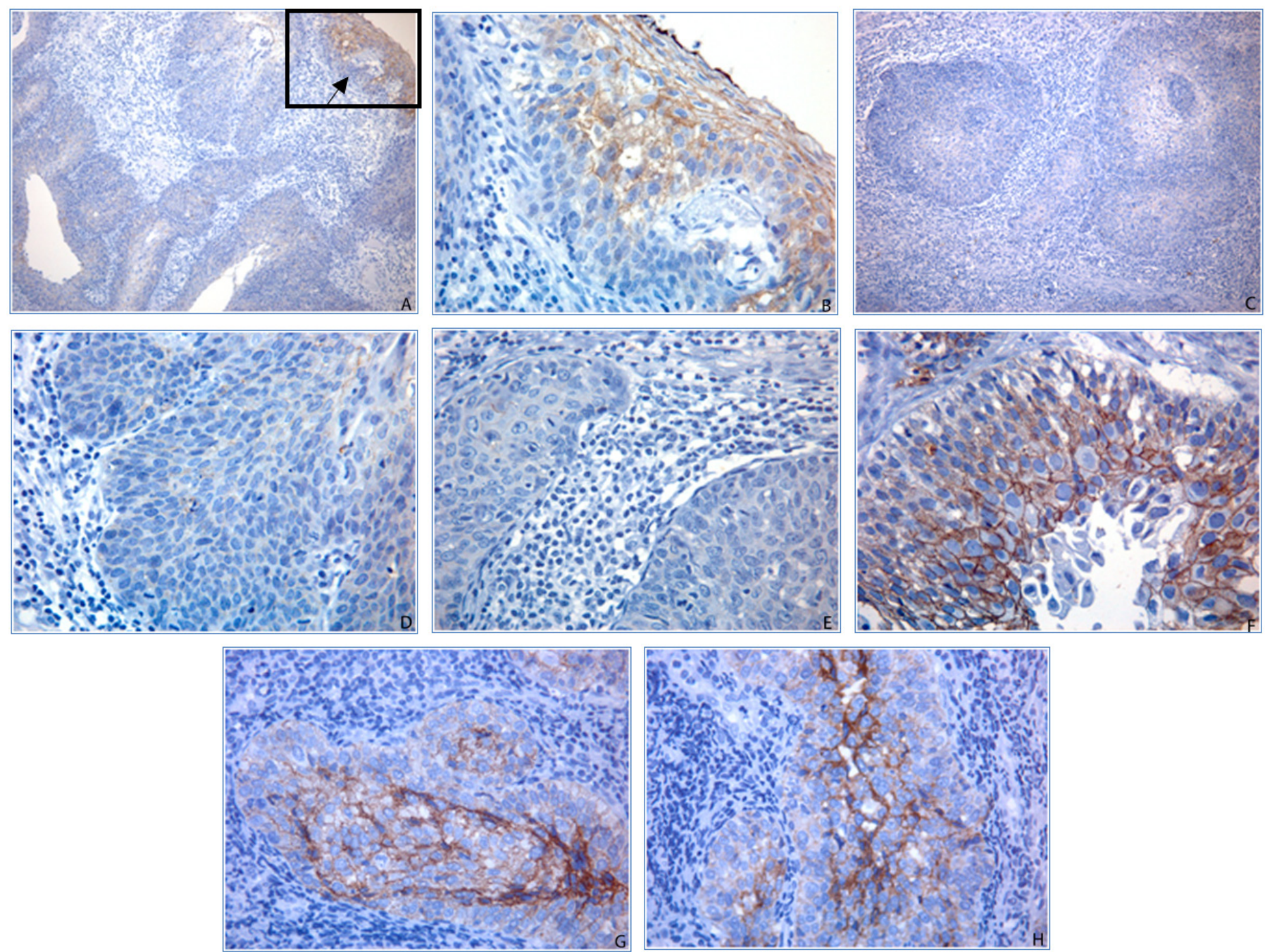

FIGURE 2 - E-cadherin immunoexpression in squamous cell carcinoma (SCC) and in HSIL: (A) SCC E-cadherin negative immunoexpression with contiguous HSIL exhibiting positive expression (arrow); (B) A higher magnification of the detailed area shown in panel A; (C) Infiltrative nests negative expression; (D) Negative expression; (E) Negative expression; (F) Infiltrative block: central area - positive expression; invasion margin - negative expression; (G) and (H) Infiltrative blocks - central area - positive expression; invasion margin - negative expression. (A and C, x100 magnification; B, D-H, x400 magnification).

Upon comparing E-cadherin negative expression between $\operatorname{SCC}(19 / 23=83 \%)$ and cervicitis $(1 / 7=12 \%)$, a highly significant difference $(\mathrm{p}=0.0009)$ was observed. In addition, a similar trend

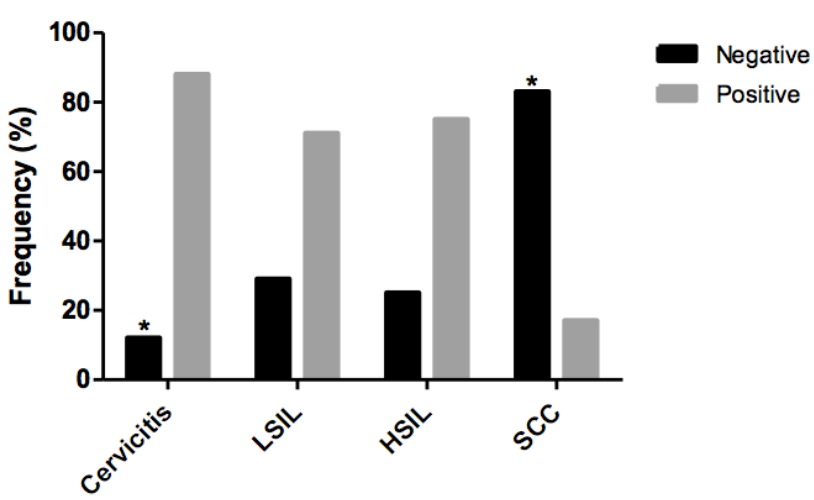

FIGURE 3 - E-cadherin immunoexpression varies among Cervicitis, LowGrade Squamous Intraepithelial Lesions (LSIL), High-Grade Squamous Intraepithelial Lesions (HSIL) and Squamous Cell Carcinoma (SCC) of the uterine cervix. ${ }^{*} \mathrm{p}=0.0009$ vs negative expression in cervicitis group was observed when we compared E-cadherin negative staining in SCCs $(19 / 23=83 \%)$ to LSILs $(7 / 24=29 \%, \mathrm{p}=0.0004)$ and to HSILs $(7 / 28=25 \%, p<0.0001)$ separately (Figure 3$)$ or when grouped together (Figure 4).

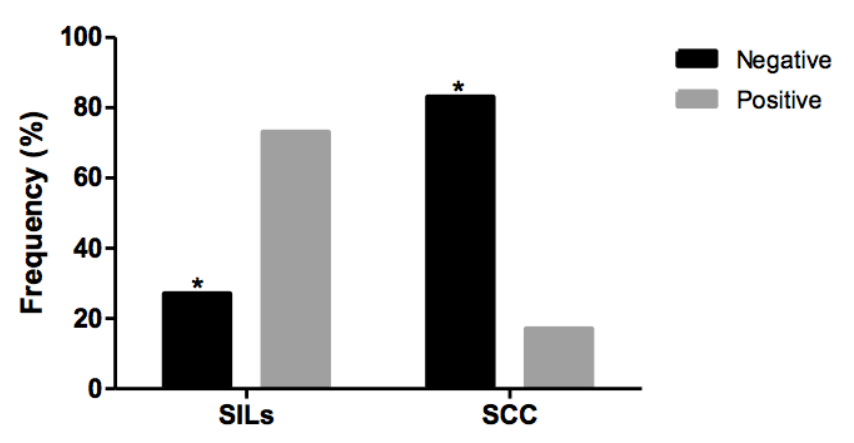

FIGURE 4 - Differential E-cadherin immunoexpression in Squamous Intraepithelial Lesions (SILs = LSILs + HSILs) and in Squamous Cell Carcinoma (SCC) of the uterine cervix. ${ }^{*} \mathrm{p}<0.0001$ SCC vs SIL negative expression 
As shown in Table 2, we observed a higher frequency of negative expression of E-cadherin in normal mucosa adjacent to the LSILs $(8 / 15=53 \%)$ and HSILs $(6 / 13=46 \%)$ than in normal mucosa adjacent to cervicitis $1 / 8(12 \%)$; however, these results did not exhibit a statistically significant difference $(\mathrm{p}=0.0858$ and $\mathrm{p}$ $=0.1736$, respectively). The negative staining pattern was similar for both types of SILs.

When comparing Tables 1 and 2, we found negative expression of E-cadherin in 7/24 LSIL cases (29\%). In contrast, negative staining was observed in $8 / 15$ cases $(53 \%, \mathrm{p}=0.1817)$ in the normal area adjacent to this lesion. Likewise, negative E-cadherin expression in HSIL was observed in 7/28 (25\%) cases, whereas negative immunoreactivity was observed in $6 / 13$ $(46 \%)$ cases in the adjacent normal tissue. However, no significant difference was observed $(\mathrm{p}=0.2797)$.

TABLE 2 - E-cadherin immunoexpression in normal epithelium adjacent to Cervicitis, Low-Grade Intraepithelial Lesions (LSIL) and High-Grade Intraepithelial Lesions (HSIL).

\begin{tabular}{lccc}
\hline LOCALIZATION & \multicolumn{2}{c}{$\begin{array}{c}\text { E-cadherin } \\
\text { immunoexpression }\end{array}$} & \\
\cline { 2 - 3 } & NOTAL & \\
\hline Adjacent to Cervicitis & $1(12 \%)$ & $7(88 \%)$ & 8 \\
Adjacent to LSIL & $8(53 \%)$ & $7(47 \%)$ & $15^{*}$ \\
Adjacent to HSIL & $6(46 \%)$ & $7(54 \%)$ & $13^{* *}$ \\
\hline
\end{tabular}

*In 9 cases the normal epithelium adjacent to LSILs was not represented.

**In 15 cases the normal epithelium adjacent to HSILs had no representation.

Table 3 shows the immunoreactivity of E-cadherin in the epithelial lining of different histological regions, including the ectocervix, squamocolumnar junctions (SCJ)/Metaplasia and the layers of epithelial thickness (1/3 basal, 1/3 intermediate, $1 / 3$ superficial), among the three groups: cervicitis, LSIL and HSIL. Predominantly negative staining was observed in the basal third of all lesions for both histological regions (ectocervix and SCJ/Metaplasia). Positive staining prevailed in the intermediate third with respect to the basal and superficial thirds in cervicitis and LSIL both in the ectocervix and in the SCJ/Metaplasia regions. The HSILs showed a different pattern in the intermediate third, with more cases of negative expression $(17 / 33=52 \%)$ when compared to the other two groups of lesions when they were considered together (11/ 40 $=28 \%)$. A marginally significant difference $(\mathrm{p}=0.0528)$ was observed in the entire sample, including both the ectocervix and $\mathrm{SCJ} /$ Metaplasia regions.

Finally, Table 4 shows the expression of E-cadherin in two distinct histological regions of the blocks formed from invasive cancer cells: the central area of the tumor cell block itself and its periphery are delineated by the invasion margin. These results predominantly show absence of expression in both regions, particularly in the margin of invasion $(22 / 23$ $=96 \%$, when compared to the agglomerates of central cells $(19 / 23=83 \%)$; however, no significant difference was observed $(\mathrm{p}=0.3463)$.

With regard to tumor budding, a detailed observation was performed on the invasion margins of our samples, but this phenomenon was not observed in our study.

TABLE 4 - E-cadherin Immunoexpression in Squamous Cell Carcinoma (SCC) of the Uterine Cervix.

\begin{tabular}{lccc}
\hline \multirow{2}{*}{ LOCALIZATION } & \multicolumn{2}{c}{ E-cadherin expression } & \multirow{2}{*}{ TOTAL } \\
\cline { 2 - 3 } & Negative & Positive & \\
\hline Central Area & $19(83 \%)$ & $4(17 \%)$ & 23 \\
Invasion Margin & $22(96 \%)$ & $1(4 \%)$ & 23 \\
\hline
\end{tabular}

TABLE 3 - E-cadherin immunoexpression in Cervicitis, Low-Grade Squamous Intraepithelial Lesions (LSIL) and High-Grade Intraepithelial Lesions (HSIL) by histological region and epithelial thickness.

\begin{tabular}{lccccccc}
\hline \multirow{2}{*}{ LOCALIZATION } & \multicolumn{6}{c}{ E-cadherin immunoexpression } \\
\cline { 3 - 7 } & & \multicolumn{2}{c}{ Cervicitis* } & \multicolumn{2}{c}{ LSIL** } & \multicolumn{2}{c}{ HSIL*** } \\
& & Negative & Positive & Negative & Positive & Negative & Positive \\
Ectocervix & Basal & 5 & 0 & 8 & 2 & 5 & 0 \\
(thirds) & Intermediate & 0 & 5 & 3 & 7 & 2 & 3 \\
& Superficial & 4 & 1 & 8 & 2 & 3 & 2 \\
SCJ/ & Basal & 5 & 1 & 15 & 4 & 22 & 6 \\
Metaplasia & Intermediate & 1 & 5 & 7 & 12 & 15 & 13 \\
(thirds) & Superficial & 6 & 0 & 11 & 8 & 13 & 15 \\
& & & & & & & \\
\hline
\end{tabular}

*Total of Cervicitis=8 (ectocervix only=2; SCJ/Metaplasia only3; ectocervix + SCJ/Metaplasia=3).

**In 14 cases there weren't lesions in ectocervix and 5 cases didn't have lesion in SCJ/Metaplasia.

***23 cases didn't show any lesion in ectocervix. 


\section{Discussion}

This study shows a progressive decrease in E-cadherin staining from SILs to SCCs, with minor expression in SILs and practically no expression in SCCs. It clearly demonstrates that SILs lose E-cadherin immunoexpression as they progress towards severity, which is in agreement with previous studies ${ }^{12,27-32}$. More interestingly, we verified that this adhesion molecule exhibits a different expression pattern according to the epithelial thickness layer.

We also observed no differences in E-cadherin negative immunoexpression in SILs. These results are similar to those of Rodriguez-Sastre et $a l .{ }^{33}$, who found that membrane E-cadherin exhibited a tendency to disappear in $40 \%$ of SILs.

The normal epithelium adjacent to the SIL areas showed greater negative E-cadherin expression than the lesions themselves. These results were unexpected, and there is no definitive answer for this observation. Despite the absence of a significant difference, it's reasonable to speculate that a specific neighboring location potentially exists that exhibits a higher potential for carcinogenesis than distant tissues. Carvalho et al. ${ }^{34}$ performed a study examining the molecular alterations in histologically negative surgical margins of head and neck cancer and found that local recurrence was apparent in approximately $20 \%$ of patients. They suggested that the intra-operative histological analysis cannot detect molecular changes that do not involve phenotypic alterations of cells, but these alterations may be moving along the tumorigenesis track. Slaughter, Southwick and Smejcal ${ }^{35}$ performed a study involving oral cancer that has called attention to what they referred to as "field cancerization" or "field of pre-conditioned epithelium" in the tumor adjacent area and suggested that such a concept would partially explain the high local recurrence rate in oral cancer.

Here, E-cadherin membrane immunoreactivity was examined in LSILs, HSILs and also in cervicitis with respect to histological region (ectocervix and SCJ/Metaplasia), and the different layers of epithelial thickness (basal, intermediate and superficial) were taken into consideration. Interestingly, these results showed predominantly negative staining in the basal third for all lesions and in both histological regions. Positive staining prevailed in the intermediate third when compared to the basal and superficial thirds in LSILs and cervicitis, in both the ectocervix and SCJ/Metaplasia regions. In contrast, HSILs exhibited a different behavior in the middle third, showing an increased negative expression for E-cadherin. It is well known that in HSIL, the less differentiated cells occupy up to two-thirds of the epithelial thickness ${ }^{36}$, and this observation would explain such a different pattern. De Boer et al. ${ }^{28}$ studied the role of cadherins and catenins during the progression of SIL in the uterine cervix and found that HSILs exhibited a strong decrease in E-cadherin expression. The progressive reduction in cell adhesion molecule (CAM) expression in neoplastic cervical tissue indicates that it may participate in the epithelial stratification process ${ }^{30}$. The lack of differentiation and epithelial organization seen in SILs may be due to disrupted expression of adhesion molecules such as E-cadherin ${ }^{27}$. Cavallaro and Christofori ${ }^{37}$ confirmed that E-cadherin plays a pivotal role in inducing cell polarity and epithelial organization.

Initially in this study, there seemed to be differences in E-cadherin staining between central cells of tumor infiltrative blocks of SCC when compared to the front periphery infiltrative cells. Thus, one goal is to verify the eventually distinct expression patterns between these two regions of infiltrative blocks. The results showed a predominance of negative expression at the invasion margin adjacent to the stroma in relation to the central areas of the infiltrative nests, but these results lacked statistical significance. This was the same conclusion made by Rodrigues et $a l .{ }^{18}$ in a similar report on vulvar carcinomas in which they did not find significant differences in E-cadherin expression between the central area and the invasion margin.

With respect to the invasion margin, which is the site of tumor budding ${ }^{19,21}$, another approach was used to look for these small cell clusters that have been recently reported elsewhere ${ }^{19-21}$. However, these buds were not observed in our samples even after a search was conducted by a pathologist specifically for this purpose. As this sample contains only well-defined invasive cancers, one possible explanation is that buds are transient and present only at the time of the carcinoma in situ to microinvasion progression. We also speculate that buds are present only at the front of the more distal cell blocks and not in the intermediate invasive ones; however, such an approach demands numerous slides obtained from hysterectomy specimens to evaluate the deep margin of the tumors, which was not part of our study design.

\section{Conclusion}

E-cadherin is an essential molecule during the process of cervical carcinogenesis and in this context exhibits a different expression pattern according to the epithelial thickness layer.

\section{Acknowledgements}

This work was supported by CNPq (Conselho Nacional de Desenvolvimento Científico e Tecnológico), CAPES 
(Coordenação de Aperfeiçoamento de Pessoal de Nível Superior). We are also grateful to the American Journal Experts for the English edition.

\section{References}

1. Globocan 2012. Estimated Cancer Incidence - Cervical Cancer Incidence, Mortality and Prevalence Worldwide in 2012 - Available from: http://globocan.iarc.fr/Pages/fact_sheets_cancer.aspx.

2. Instituto Nacional de Câncer José Alencar Gomes da Silva (INCA). Estimativa 2014 - Incidência de Câncer no Brasil - Available from: http://www.inca.gov.br/estimativa/2014/.

3. Meijer CJ, Snijders PJ, Castle PE. Clinical utility of HPV genotyping. Gynecol Oncol. 2006 Oct; 103(1): 12-7. doi: 10.1016/j. ygyno.2006.07.031.

4. Walboomers JMM, Jacobs MV, Manos MM, Bosch FX, Kummer JA, Shah KV, Snijders PJF, Peto J, Meijer CJL, Muñoz N. Human papillomavirus is a necessary cause of invasive cervical cancer worldwide. J Pathol. 1999 Sep;189(1):12-9. PMID: 10451482.

5. Lee MY, Shen MR. Epithelial-mesenchymal transition in cervical carcinoma. Am J Transl Res. 2012;4(1):1-13. PMID: 22347518.

6. Szalmás A, Kónya J. Epigenetic alterations in cervical carcinogenesis. Semin Cancer Biol. 2009 Jun;19(3):144-52. doi: 10.1016/j.semcancer.2009.02.011.

7. Richart RM. Natural history of cervical intraepithelial neoplasia. Clin Obstet Gynecol. 1967 Dec;10(4):748-84. PMID: 8463044.

8. Snijders PJF, Steenbergen RDM, Heideman DAM, Meijer CJLM. HPVmediated cervical carcinogenesis: concepts and clinical implications. J Pathol. 2006 Jan;208(2):152-64. doi: 10.1002/path.1866.

9. Waxman AG, Chelmow D, Darragh T, Lawson H, Moscicki AB. Revised terminology for cervical histopathology and its implications for management of high-grade squamous intraepithelial lesions of the cervix. Obstet Gynecol. 2012 Dec;120(6):1465-71. doi: http://10.1097/AOG.0b013e31827001d5.

10. Hanahan D, Weinberg RA. The hallmarks of cancer. Cell. 2000 Jan 7;100(1):57-70. PMID: 10647931.

11. Hay ED. The mesenchymal cell, its role in the embryo, and the remarkable signaling mechanisms that create it. Dev Dyn. 2005;233:706-20. doi: 10.1002/dvdy.20345.

12. Caberg JHD, Hubert PM, Begon DY, Herfs MF, Roncarati PJ, Boniver JJ, Delvenne PO. Silencing of E7 oncogene restores functional E-cadherin expression in human papilomavírus 16-transformed keratinocytes. Carcinogenesis. 2008 Jul;29(7):1441-7. doi: 10.1093/ carcin/bgn145.

13. Jodele S, Blavier L, Yoon JM, DeClerck YA. Modifying the soil to affect the seed: role of stromal derived matrix metallproteinases in cancer progression. Cancer Metastasis Rev. 2006;25:35-43. doi: 10.1007/s10555-006-7887-8.

14. Behrens J, Mareel MM, Van Roy FM, Birchmeier W. Dissecting tumor cell invasion: epithelial cells acquire invasive properties after the loss of uvomorulin-mediated cell-cell adhesion. J Cell Biol. 1989 Jun;108(6):2435-47. PMID: 2661563.

15. Burdsal CA, Damsky CH, Pedersen RA. The role of E-cadherin and integrinas in mesoderm differentiation and migration at the mammalian primitive streak. Development. 1993 Jul;118(3):82944. PMID: 7521282.

16. Katto J, Mahlknecht U. Epigenetic regulation of cellular adhesion in cancer. Carcinogenesis. 2011 Oct;32(10):1414-8. doi: 10.1093/ carcin/bgr120.

17. Shinto E, Mochizuki H, Ueno H, Matsubara O, Jass JR. A novel classification of tumour budding in colorectal cancer based on the presence of cytoplasmic pseudo-fragments around budding foci. Histopathology. 2005 Jul;47(1):25-31. doi: 10.1111/j.13652559.2005.02162.x.

18. Rodrigues LS, Lavorato-Rocha AM, Maia BM, Stiepcich MMA, Carvalho FM, Baiocchi G, Soares FA, Rocha RM. Epithelialmesenchymal transition-like events in vulvar cancer and its relation with HPV. Br J Cancer. 2013 Jul 9;109(1):184-94. doi: 10.1038/ bjc.2013.273.

19. Okuyama T, Oya M, Yamaguchi M. Budding (sprouting) as a useful prognostic marker in colorectal mucinous carcinoma. Jpn J Clin Oncol. 2002 Oct;32(10):412-6. PMID: 12451038.

20. Hase K, Shatney C, Johnson D, Trollope M, Vierra M. Prognostic value of tumor "budding" in patients with colorectal cancer. Dis Colon Rectum. 1993 Jul;36(7):627-34. PMID: 8348847.

21. Jass JR, Barker M, Fraser L, Walsh MD, Whitehall VLJ, Gabielli B, Young J, Leggett JBA. APC mutation and tumour budding in colorectal cancer. J Clin Pathol. 2003 Jan;56(1):69-73. PMID: 12499439.

22. Karamitopoulou E. Role of epithelial-mesenchymal transition in pancreatic ductal adenocarcinoma: is tumor budding the missing link? Front Oncol. 2013 Sep 17;3:1-5. doi: 10.3389/fonc.2013.00221.

23. Hunt NCA, Douglas-Jones AG, Jasani B, Morgan JM, Pignatelli M. Loss of E-cadherin expression associated with lymph node metastasis in small breast carcinomas. Virchows Arch. 1997;430:285-9. PMID: 91340339.

24. Jawhari A, Jordan S, Poole S, Browne P, Pignatelli M, Farthing MJG. Abnormal immunoreactivity of the E-cadherin-catenin complex in gastric carcinoma: relationship with patient survival. Gastroenterology. 1997 Jan;112(1):46-54. PMID: 16231374.

25. Shimazui T, Kojima T, Onozawa M, Suzuk M, Asano T, Akaza $\mathrm{H}$. Expression profile of N-cadherin differs from other classical cadherins as a prognostic marker in renal cell carcinoma. Oncol Rep. 2006 May;15(5):1181-4. PMID: 16596183.

26. Almeida PR, Ferreira VA, Santos CC, Rocha-Filho FD, Feitosa RR, Falcão EAA, Cavada BK, Ribeiro RA. E-cadherin immunoexpression patterns in the characterization of gastric carcinoma histotypes. J Clin Pathol. 2010 Jul;63(7):635-9. doi: 10.1136/jcp.2010.076026.

27. Carico E, Atlante M, Bucci B, Nofroni I, Vecchione A. E-cadherin and $\alpha$-catenin expression during tumor progression of cervical carcinoma. Gynecol Oncol. 2001 Feb;80(2):156-61. doi: 10.1006/ gyno.2000.6035

28. De Boer CJ, E. van Dorst E, van Krieken H, Jansen-van Rhijn CM, Warnaar SO, Fleuren GJ, Litvinov SV. Changing roles of cadherins and catenins during progression of squamous intraepithelial lesions in the uterine cervix. Am J Pathol. 1999 Aug;155(2):505-15. PMID: 10433943.

29. Hubert P, Caberg JH, Gilles C, Bousarghin Franzen-Detrooz LE, Boniver J, Delvenne P. E-cadherin-dependent adhesion of dendritic and Langerhans cells to keratinocytes is defective in cervical human papilomavírus-associated (pre) neoplastic lesions. J Pathol. 2005 Jul;206(3):346-55. doi: 10.1002/path.1771.

30. Mendez MT, Bosch AL. Abnormal immunoexpression of cell adhesion molecules (cams) in cervical cancer. Int J Surg Pathol. 2011;19:733-42. doi: 10.1177/1066896909343435.

31. Munhoz NG, Rodrigues DA, Pedregosa JF, Rodrigues JO, Junqueira MSG, Yonamine PTK, Pereira SF, Uezato S, Pandossio T, Martins EKL, Oliveira FB, Cordeiro JA, Bonilha JL, Cury PM. The Use of molecular markers (p16, Ki-67 and E-cadherin) in uterine cervical biopsies. Open Pathol J. 2009;3:10-7. doi: 10.2174/1874375700903010010.

32. Wu Y, Zhou BP. New insights of epithelial-mesenchymal transition in cancer metastasis. Acta Biochim Biophys Sin. 2008 Jul;40(7):64350. doi: 10.1111/j.1745-7270.2008.00443.x.

33. Rodríguez-Sastre MA, González-Maya L, Delgado R, Lizano M, Tsubaki G, Mohar A, García-Carrancá A. Abnormal distribution of 
Cavalcante JR et al.

E-cadherin and $\beta$-catenin in different histologic types of cancer of the uterine cervix. Gynecol Oncol. 2005;97:330-36. doi: 10.1016/j. ygyno.2004.12.062.

34. Carvalho AC, Kowalski LP, Campos AHJFM, Soares FA, Carvalho $\mathrm{AL}$, Vettore AL. Clinical significance of molecular alterations in histologically negative surgical margins of head and neck cancer patients. Oral Oncol. 2012 Mar;48(3):240-8. doi: 10.1016/j. oraloncology.2011.10.018.

35. Slaughter DP, Southwick HW, Smejcal W. "Field cancerization" in oral stratified squamous epithelium - Clinical implications of multicentric origin. Cancer. 1953 Sep;6(5):963-8. PMID: 13094644.

36. Buckley $\mathrm{CH}$, Butler EB, Fox H. Cervical intraepithelial neoplasia. J Clin Pathol. 1982 Jan;35(1):1-13. doi: 10.1136/jcp.35.1.1

37. Cavallaro U, Christofori G. Cell adhesion and signaling by cadherins and IG-CAMS in cancer. Nat Rev Cancer. 2004 Feb;4(2):118-32. doi: $10.1038 / \mathrm{nrc} 1276$.

\section{Correspondence:}

Paulo Roberto Carvalho Almeida

Departamento de Patologia e Medicina Legal/FAMED/UFC

Rua Monsenhor Furtado, S/N

60.430-350 Fortaleza-CE Brasil

Tel.: (55 85)3366-8307

Fax: (55 85)3366-8316

paulo.almeida@ufc.br zeruso@yahoo.com.br zerusocaval@gmail.com

Received: May 19, 2014

Review: July 18, 2014

Accepted: Aug 22, 2014

Conflict of interest: none

Financial source: none

${ }^{1}$ Research performed at Laboratory of Pathology, Department of Pathology and Forensic Medicine, Federal University of Ceara (UFC), Fortaleza-CE, Brazil. Part of Master degree thesis, Postgraduate Program in Pathology, UFC. Tutor: Paulo Roberto Carvalho Almeida. 